\title{
Ressecção Pulmonar: Análise do Banco de Dados da Sociedade Brasileira de Cirurgia Torácica
}

\author{
Pulmonary Resection: Analysis of the Database of the \\ Brazilian Society of Thoracic Surgery
}

\begin{abstract}
Ricardo M. Terra ${ }^{1}$, Maria Teresa Ruiz Tsukazan ${ }^{2}$, Gustavo Fortunato ${ }^{3}$, Spencer M. Camargo $^{4}$, Leticia Lauricella ${ }^{5}$, Humberto A. De Oliveira ${ }^{6}$, Darcy R. Pinto ${ }^{7}$ ${ }^{1}$ Serviço de Cirurgia Torácica do Hospital das Clínicas da FMUSP; São Paulo, São Paulo; ${ }^{2}$ Serviço de Cirurgia Torácica do HSL-PUCRS; Porto Alegre, Rio Grande do

Sul; ${ }^{3}$ Serviço de Cirurgia Torácica do Hospital Santa Izabel; Salvador, Bahia, ${ }^{4}$ Serviço de Cirurgia do Hospital Geral de Caxias do Sul; Caxias do Sul, Rio Grande do Sul; ${ }^{5}$ Serviço de Cirurgia Torácica do Hospital das Clínicas da FMUSP; ${ }^{6}$ Serviço

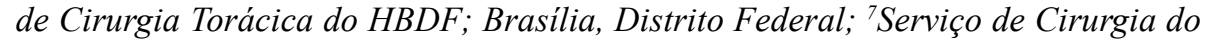
Hospital Geral de Caxias do Sul; Brasil
\end{abstract}

Correspondence addresses: Dr. Gustavo Fortunato gustavofortunato@cirtoraxbahia.com.br

Received: February 12, 2019

Revised: March 14, 2019

Accepted: March 12, 2019

Published: March 27, 2019

Data Availability Statement: All relevant data are within the paper and its Supporting Information files.

Competing interests: The authors have declared that no competing interests exist.

\section{Copyright}

(C) 2019 by Santa Casa de Misericórdia da Bahia. All rights reserved.

ISSN: 2526-5563
A Sociedade Brasileira de Cirurgia Torácica (SBCT) utiliza uma versão customizada da plataforma de banco de dados da Sociedade Europeia de Cirurgia Torácica (ESTS). Nesta análise, de agosto/15 a dezembro/ 15, 1.367 pacientes haviam sido registrados no banco de dados. Destes, 902 haviam sido submetidos à ressecção pulmonar anatômica (lobectomia, segmentectomia ou pneumectomia). O desfecho analisado foi morbi-mortalidade intrahospitalar.

A média de idade foi 59,6 anos $( \pm 15,2)$ e $52,5 \%$ eram mulheres. O diagnóstico de base (n=597) foi câncer de pulmão em $450(75,3 \%)$, bronquiectasia ou malformação pulmonar em 70 (11,7\%), destruição pulmonar secundária a tuberculose em $57(9,5 \%)$ e metástase em 14 (2,4\%). O escore da American Society of Anesthesiology (ASA) ( $\mathrm{n}=793$ )

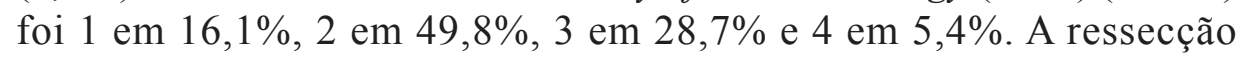
mais realizada foi lobectomia, com 681 casos, $75,5 \%$, das quais $45 \%$ foram por videotoracoscopia (VATS), pneumectomia 71 (7,9\% - 13\% VATS), bilobectomia 39 (4,3\%-13\% VATS) e segmentectomia 111 (12,3\%-66\% VATS).

O índice de morbidade foi de $36,7 \%$ e isto variou de acordo com o procedimento realizado. O perfil de complicação também variou entre lobectomia e pneumectomia (Tabela 1). O índice de mortalidade global foi de $2,6 \%(22 / 843)$, variando conforme o procedimento: lobectomia $11 / 641$ $(1,7 \%)$, pneumectomia $6 / 62(8,8 \%)$, bilobectomia $2 / 36(5,5 \%)$ e segmentectomia 3/103 (2,8\%). As complicações mais relevantes com evolução fatal foram pneumonia (11), infarto do miocárdio (3), sangramento requerendo reoperação (1) e impossibilidade de desmame da ventilação (1). 
Tabela 1. Perfil de morbidade.

\begin{tabular}{lccc}
\hline Complicações & $\begin{array}{c}\text { Todos pacientes } \\
\mathbf{N}=\mathbf{7 5 3}\end{array}$ & $\begin{array}{c}\text { Lobectomia } \\
\mathbf{N}=\mathbf{5 6 2}\end{array}$ & $\begin{array}{c}\text { Pneumectomia } \\
\mathbf{N}=\mathbf{6 3}\end{array}$ \\
\hline Complicações cardiopulmonares maiores & $146(19,4 \%)$ & $113(20 \%)$ & $13(20,7 \%)$ \\
Pneumonia & $82(10,9 \%)$ & $64(11,4 \%)$ & $3(7,9 \%)$ \\
Escape aéreo >5 dias & $61(8,1 \%)$ & $46(8,2 \%)$ & $3(4,8 \%)$ \\
Atelectasia & $41(5,4 \%)$ & $36(6,4 \%)$ & - \\
Arritmia atrial & $29(3,9 \%)$ & $20(3,6 \%)$ & $3(4,8 \%)$ \\
Delirium & $19(2,5 \%)$ & $14(2,5 \%)$ & $2(3,2 \%)$ \\
Empiema & $14(1,9 \%)$ & $4(0,7 \%)$ & $6(9,5 \%)$ \\
Insuficiência renal & $12(1,6 \%)$ & $10(1,9 \%)$ & $1(1,6 \%)$ \\
Infarto do miocárdio & $11(1,5 \%)$ & $8(1,4 \%)$ & $2(3,2 \%)$ \\
Embolia pulmonar & $10(1,3 \%)$ & $9(1,6 \%)$ & $1(1,6 \%)$ \\
Reoperação por sangramento & $8(1,1 \%)$ & $5(0,9 \%)$ & - \\
Reintubação & $8(1,1 \%)$ & $5(0,9 \%)$ & $1(1,6 \%)$ \\
Fístula broncopleural & $5(0,7 \%)$ & $3(0,5 \%)$ & $1(1,6 \%)$ \\
Traqueostomia & $4(0,5 \%)$ & $3(0,5 \%)$ & - \\
Infecção de ferida & $3(0,4 \%)$ & - & - \\
SARA & $3(0,4 \%)$ & $1(0,2 \%)$ & - \\
Ventilação inicial > 48h & $3(0,4 \%)$ & $2(0,4 \%)$ & - \\
Complicações cerebro-vasculares & $1(0,1 \%)$ & $1(0,2 \%)$ & - \\
Edema pulmonar & $1(0,1 \%)$ & - & $1(1,6 \%)$ \\
Outros & $93(12,3 \%)$ & $63(12 \%)$ & $17(27 \%)$ \\
Qualquer complicação & $277(36,7 \%)$ & $207(36,8 \%)$ & $28(44,4 \%)$ \\
\hline
\end{tabular}

